Modern Physics Letters A,

(C) World Scientific Publishing Company

\title{
ANISOTROPY IN THE PROPAGATION OF RADIO POLARIZATIONS FROM COSMOLOGICALLY DISTANT GALAXIES
}

\author{
PANKAJ JAIN \\ Physics Department, I.I.T. Kanpur \\ Kanpur, India - 208016 \\ JOHN P. RALSTON \\ Department of Physics and Astronomy, Kansas University \\ Lawrence, KS-66045, USA
}

Received (received date)

Revised (revised date)

\begin{abstract}
Radiation traversing the observable universe provides powerful ways to probe anisotropy of electromagnetic propagation. A controversial recent study claimed a signal of dipole character. Here we test a new and independent data set of 361 points under the null proposal of statistical independence of linear polarization alignments relative to galaxy axes, versus angular positions. The null hypothesis is tested via maximum likelihood analysis of best fits among numerous independent types of factored distributions. We also examine single-number correlations which are parameter free, invariant under coordinate transformations, and distributed very robustly. The statistics are shown explicitly not to depend on the uneven distribution of sources on the sky. We find that the null proposal is not supported at the level of less than $5 \%$ to less than $0.1 \%$ by several independent statistics. The signal of correlation violates parity, that is, symmetry under spatial inversion, and requires a statistic which transforms properly. The data indicate an axis of correlation, on the basis of likelihood determined to be $\left[\right.$ R.A. $=\left(0^{\mathrm{h}}, 9^{\mathrm{m}}\right) \pm\left(1^{\mathrm{h}}, 0^{\mathrm{m}}\right)$, Decl. $\left.=-1^{\circ} \pm 15^{\circ}\right]$.
\end{abstract}

\section{Introduction}

The orientation of linear radio polarizations emitted by cosmologically distant galaxies has a consistent relation with the galaxy symmetry axis. Exceedingly small physical effects accumulate during propagation, which conventional measurements can directly probe. Thus electromagnetic radiation traversing the observable universe can detect subtle forms of cosmological anisotropy. A signal with dipole character was claimed recently ${ }^{1}$ from an analysis of published radio data. Analysis found an "anisotropy axis" $\vec{s}_{\mathrm{NR}}=\left(21^{\mathrm{h}} \pm 2^{\mathrm{h}}, 0^{\circ} \pm 20^{\circ}\right)$ governing orientation of polarization of the radio signals varying in a coherent way across the dome of the sky. The origin of this behavior is not clear, and may or may not indicate a fundamental anisotropy on a scale larger than previously found in cosmology.

There is a long history of puzzling observations. Beginning in the 1960's observers noticed that Faraday-subtracted polarizations were distributed in peculiar ways relative to the source axes. In $1982 \mathrm{Birch}^{2}$ empirically observed a coherent an- 
gular anisotropy in the off-sets of the polarization and galaxy axes, using a data set of 137 points. Birch's statistical methods were questioned, but more sophisticated studies $^{3,4}$ confirmed surprisingly strong signals in Birch's data. The statistics were not consistent with isotropy at $99.9 \%$ and $99.98 \%$ confidence levels, respectively. One of the same groups ${ }^{4,5}$ went on to create an independent set of 277 points and simultaneously introduced a different statistical measure. They obtained no signal in this set and dismissed Birch's results. This left unresolved the puzzling fact that his data had contained a signal at such a high level of statistical significance. When Nodland and Ralston, ${ }^{1}$ initially unaware of Birch's ${ }^{2}$ work, independently found a statistically significant signal in an independent set of 160 points, criticisms focused on proposing different statistical baselines ${ }^{6,7,8}$ and again claimed to find no signal of anisotropy. The question of systematic bias in such data had been raised by the authors $^{1}$ (henceforth $N R$ ) and earlier ${ }^{9}$ regarding Birch.

Here we report analysis of a considerably larger data set which contains 361 points. We have taken into account criticisms and experience from earlier work, and used the most robust statistical methods available. New progress has been made by paying close attention to the symmetries of the problem. The usual expectation of independence of the polarization and sky angular coordinates, or "uncorrelated isotropy", happens to represent a definite symmetry, which is that the distribution factors. The classic scientific method becomes applicable: we can test isotropy as a clean hypothesis and see if it can be ruled out, which is immensely powerful. We use generic methods to represent the correlations, emphasising the symmetry that they are odd in the polarization variable at hand, which is a consequence of parity (spatial inversion) symmetry. ${ }^{10}$ This simple point resolves many apparent discrepancies between the previous studies. Rather than being at odds with one another, all the facts are now found to be consistent; we know of nothing in contradiction to our conclusions.

The data collects variables from cosmologically distant galaxies, as compiled in the literature. ${ }^{2,4,5,11,12,13}$ The data set by $N R$ reproduced that of Carroll et al ${ }^{13}$ except for a half-dozen corrections from the original literature. The compilation of Eichendorf and Reinhardt, ${ }^{11,12}$ available on the NASA-ADC archives, contain numerous sources for which the position angle of the source is listed. We obtained the polarization angle for these sources from Simard-Normandin et $\mathrm{al}^{14}$ for all the sources for which they were available. We compiled a total of 152 data points in this fashion. Taking these as our primary data set we added any distinct data points contained in Bietenholz ${ }^{5}$, making a total of 313 points. Data points were regarded as distinct if they had different Right Ascension, and differed in Declination by more than one degree (which can be attributed to change in convention). This set was further combined with the $N R$ and remaining distinct points of the Birch data, in that order, making a total of 361 data points. In combining these different data sets, we verified that the polarization off-set values for points with coincident Right Ascension and Declination did not differ by more than a few degrees for most of the data. Specifically we found that the disagreement exceeded $5^{\circ}$ only for very 
(a)
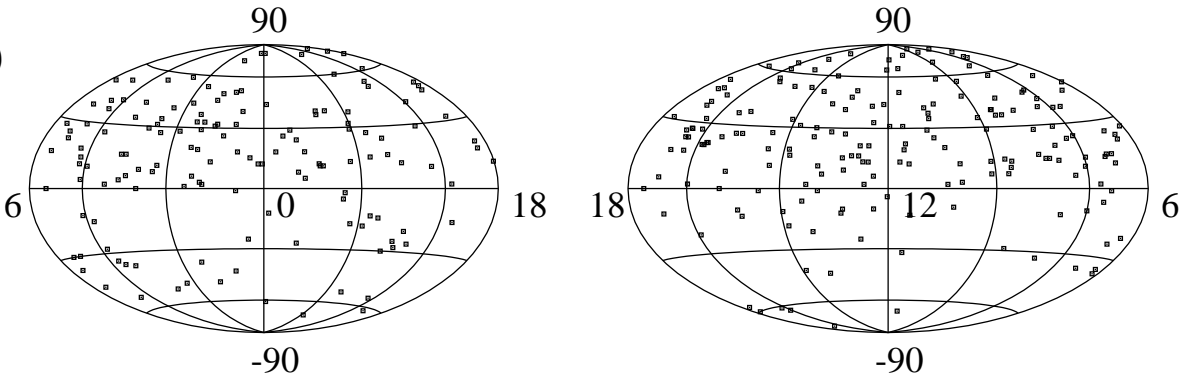

(b)
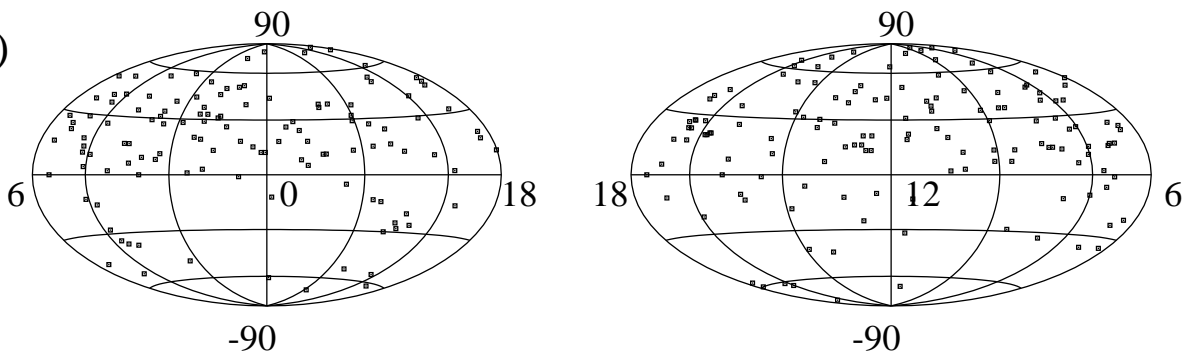

Figure 1: Aithoff-Hammer equal-area plots of the distribution of sources on the dome of the sky, in the equatorial coordinates of the data used. The distribution is somewhat non-uniform due to the zone of avoidance and dominance of Northern Hemisphere measurements. (a) The distribution of the full data set of 332 points, excluding the 29 extra points contained in Birch's compilation. Adding Birch's data makes the set even more uniform. (b) The distribution of the same data set after the cut on rotation measure, $|R M-\overline{R M}|>6$. Any non-uniformity of the angular distribution is taken into account in all statistics reported.

few points, which if deleted made no difference to our final results. We also verified consistency using a newer 1988 compilation by Broten et al. ${ }^{15}$ The only exception to this rule was found for Birch's data: here the disagreement with other compilations was found to be larger, but still tolerable. All results we report are consistent, and no combination of any large set gave results significantly different from any other. The absence of information available to us on Birch's $R M$ values, plus the possibility of discrepancies in that data, led us to give results both with and without Birch's data. In Figure 1 we show the angular distribution of data, which naturally is not isotropic due to the zone of avoidance and dominance of Northern Hemisphere measurements. We will exhaustively show that the angular distribution is not an issue and cannot be confused with correlation.

The observables listed for galaxy $i$ include a major axis orientation angle $\psi_{i}$, a linear polarization angle $\chi_{i}$, and the angular coordinates of the galaxies on the sky. Other variables may include a resolution parameter, degree of polarization, and the Faraday rotation measure $R M$. The rotation measure is the slope of plots of 
measured polarization angle versus wavelength-squared. This is known to measure intervening magnetized plasma parameters. A-priori, $R M$ has nothing to do with the variable $\chi$, which is the polarization angle after Faraday rotation is subtracted. However we have retained this variable, which seems to be informative. Consistent with restricting the study to uncorrelated isotropy, we integrate over the redshift, which happens to be incomplete in the data set in any event. We let $\beta=\chi-\psi$ be the angle between the plane of polarization and the symmetry axis of the source. The variables $\chi$ and $\psi$ are determined up to a multiple of $\pi ; \beta$ runs from $-\pi$ to $\pi .{ }^{10}$ To deal with the $\pi$ ambiguity of polarization and axis measurements, one can map $\beta \rightarrow Y(\Omega)$, where $\Omega$ is a variable defined on twice the interval. A popular map is "Map 1", $\Omega_{1}(\beta)=2 \beta$. The function $Y$ is represented by a Fourier series with periodicity $2 \pi$, assuring that the transformation $\beta \rightarrow \beta^{\prime}=\beta \pm \pi$ leaves $Y(\Omega)$ invariant. The first Fourier components create a 2 -component vector-like object $\vec{Y}(\Omega)=(\cos (\Omega), \sin (\Omega))$. When the components of $\vec{Y}(\Omega)$ are used in statistical analysis, there is naturally a Jacobian factor which represents the choice of Map. By no means, then, is Map 1 sacred, and other maps are discussed below. The angular positions on the dome of the sky are mapped into their 3-dimensional Cartesian vector positions $\vec{X}$ on a unit sphere. Since we do not model this distribution, but take it from the data, this standard map is adequate. When coordinate origins are changed, the components of $\vec{X}$ transform by standard rules; one can go on to make nicely transforming distributions and tensor correlations. The two choices measuring $\chi$ relative to $\psi$ or $\psi+\pi / 2$ correspond to $\vec{Y} \rightarrow-\vec{Y}$. This does not mix the 2 components of $\vec{Y}$, which will be called "even" (for $\cos (\Omega)$ ) and "odd" (for $\sin (\Omega)$ ) following the transformation property of being even or odd, respectively, under parity (spatial inversion). As discussed in detail elsewhere,${ }^{10}$ functions of the offset angles have the corresponding parity if they are even or odd functions of $\beta$, as intuitively evident from the handed "sense of twist" a parity-odd quantity conveys. The invariant correlations discussed below avoid any question of coordinate origin (either in polarization quantities or in angular positions on the dome of the sky) by being totally independent of the choice of angular origin.

The standard assumption of statistical independence corresponds to a distribution $g(\Omega, \vec{X})=h(\Omega) f(\vec{X})$. This is a very broad class of distributions, with $h(\Omega)$ and $f(\vec{X})$ completely unrestricted, which nevertheless has symmetries allowing it to be tested. All statistics will be compared to baselines using the actual distribution of the data $f(\vec{X})$ on the dome of the sky in Monte Carlo simulations. Statistics based on assuming independence of polarizations and positions will be compared with a

simple correlated ansatz of the form $h(\Omega) C(\Omega, \vec{X}) f(\vec{X})$. The case $C=1$ reduces to the uncorrelated case.

\section{Methods}

We report both raw statistics and also "P-values", defined as the integrated probability for the null proposal to give an equal or larger statistic. We also use "confidence levels" defined to be 1-P. We will present 2 methods testing for independence: 


\subsection{Invariant Correlations}

"Summary" correlations are single number quantities which do not necessarily probe all types of relationship. We use these for preliminary tests, and to support the more sensitive likelihood analysis with complementary information.

Following Jupp and Mardia ${ }^{16}(J M)$, multivariable $p \times q$ matrices are defined via $m_{i j}^{X Y}=\sum_{\ell}(X-\bar{X})_{i}^{\ell}(Y-\bar{Y})_{j}^{\ell}$, with $\left(m^{X X}\right)_{i j}^{-1}$ etc. similarly defined. Note that all vectors used in the correlation matrices have their means in the sample, denoted by "bar", subtracted. The invariant JM correlation test statistics for $n$ data points are $n \rho_{p \times q}^{2}$ where $\rho_{p \times q}^{2}=\operatorname{Tr}\left[\left(m^{X X}\right)^{-1} \cdot\left(m^{X Y}\right) \cdot\left(m^{Y Y}\right)^{-1} \cdot m^{Y X}\right]$ where $\operatorname{Tr}$ denoted the trace of the matrix. An important and simple feature of $\rho_{p \times q}^{2}$ is that $\rho_{p \times q}^{2}=0$ when the distribution (of any kind) is uncorrelated. The $J M$ correlations, in addition, satisfy $0 \leq \rho^{2} \leq \min (p, q)$, achieving maximum for perfect correlation, and distribution of $\rho_{p \times q}^{2}$ independent of the marginal distributions. Note that $\rho_{p \times q}^{2}$ is invariant under separate rotations of origins of galaxy axes and polarizations, or orthogonal transformations of sky coordinates, and also does not involve any parameters. We have $p=3, q=1$ for the correlation of sky positions $\vec{X}$ and the separate evenor odd-parity ${ }^{10} \beta$ representations $\vec{Y}_{1}, \vec{Y}_{2}$, respectively. The distribution of $n \rho_{3 \times 1}^{2}$ is known to be $\chi_{3}^{2}$ for large $n$. We verified this with extensive Monte Carlo simulations, confirming that the statistics are quite robust. In fact, the statistics of $n \rho^{2}$ were devoid of detectable dependence on different marginal distributions, which included the flat distribution, the von Mises, and shuffled distributions described below. A graph of Monte Carlo generated distributions from 10, 000 trials is shown in Figure 2, showing excellent agreement with the $\chi_{3}^{2}$ distribution, while consistency of $\mathrm{P}$-values was also checked to eliminate the possibility of long tails.

The $J M$ correlations are simple canonical summaries inspired by Gaussian statistics. For angular variables they are limited, but suited to sense particular linear relations between $\vec{X}$ and $\vec{Y}$, namely orthogonal rotations times projections. ${ }^{10}$ Other natural coherent correlations can unfortunately yield zero - for example, the $J M$ correlation fails to see that vector $\hat{\theta}$ in spherical polar coordinates is correlated with a uniform distribution of angular position. Such limitations are expected because correlation might take myriad forms orthogonal to a summary statistic's power to probe. A small $J M$ test correlation does not prove isotropy for this reason. Nevertheless a sufficiently large $J M$ correlation can logically rule out isotropy with a definite statistical significance.

\subsection{Independence of Statistics on the Sky Distribution}

Not only are the JM statistics independent of the distribution of $\beta$, but for large $N_{\text {data }}$ also independent of the distribution of data points on the dome of the sky. This is because they are tests for independence, not tests of particular distributions. The formal proof is given by JM. ${ }^{16}$ We also tested the distribution directly with the data and with Monte Carlo trials. Specifically (Figure 2), we shuffled the full 
(a)

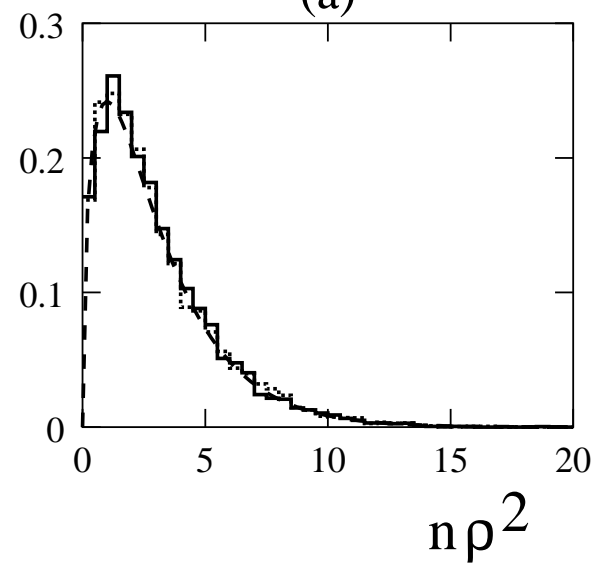

(b)

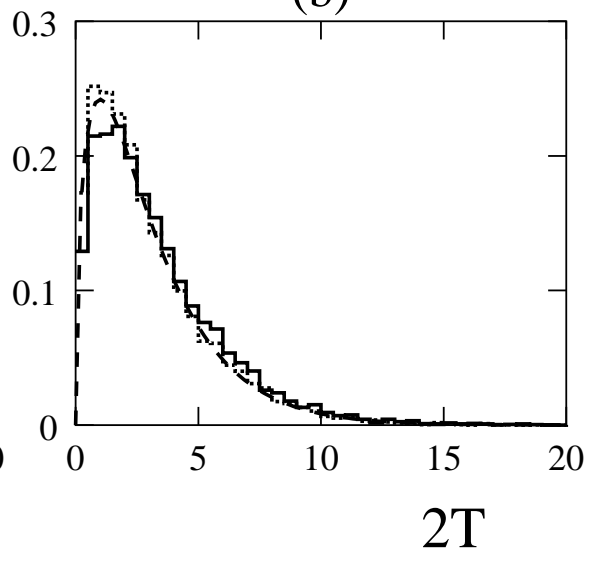

Figure 2: (a) Distributions of the $1 \times 3 \mathrm{JM}$ statistic discussed in the text. Dashed curve: the distribution $\chi_{3}^{2}$ predicted by JM. Solid histogram: the distribution generated by shuffling the full data set's $\beta$ and $\vec{X}$ coordinates to create an uncorellated null distribution with the same marginal $\beta$ and $\vec{X}$ distributions. Dotted histogram: same as the dashed case, but with the data set cut to $|R M-\overline{R M}|>6$. The Monte Carlo distributions are indistinguishable from the analytic prediction, and demonstrate that the statistic does not depend on the distribution of points on the sky. (b) Distribution of the statistic $2 T$, where $T$ is the difference of likelihoods of the correlated model and the null model. The dashed curve is the distributions $c h i_{3}^{2}$ predicted on the basis of asymptotic theory. The solid histogram is the result obtained by Monte Carlo simulation for the case of the full data set of 332 points. The dotted histogram is the result of the simulation obtained after the cut $|R M-\overline{R M}|>6$. The $\beta$ values were generated by shuffling the $\beta$ values of the original measured data sets. For the case of the cut $|R M-\overline{R M}|$, it was found that in no cases (either for the Jupp Mardia analysis and the Likelihood analysis) did $\mathrm{P}$-values generated by the Monte Carlo using 10,000 random sets exceed P-values reported, a consistency check eliminating any long tails.

data sets $\beta$ and $\vec{X}$ values to create an uncorrelated null distribution having the same marginal distributions in $\beta$ and $\vec{X}$ as the data. Shuffling is preferred here, eliminating any question of possible imperfections of models of the uneven sky distribution, which were never used anywhere in our analysis. Runs with 10, 000 copies of shuffled data gave excellent agreement with the analytic distribution known to be $\chi_{3}^{2}$. Next we repeated the procedure with the large $R M$ cut, to be discussed. The distribution was again the same (Figure 2) and in excellent agreement. This is another demonstration that there is no issue of the non-uniformity of the sky distribution affecting the statistics. There have been many misunderstandings of this basic point. We went on to try an experiment with the same number points, and using the $\beta$ values from the data, but with $\vec{X}$ values randomly distributed in extremely restricted angular regions, within a cluster of of half-angle \pm 7.5 degrees 
(a)
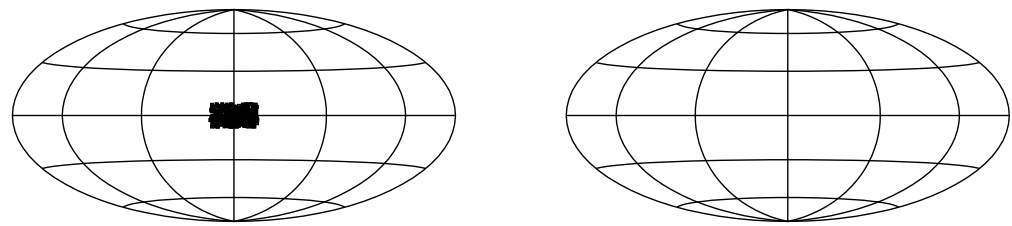

(b)
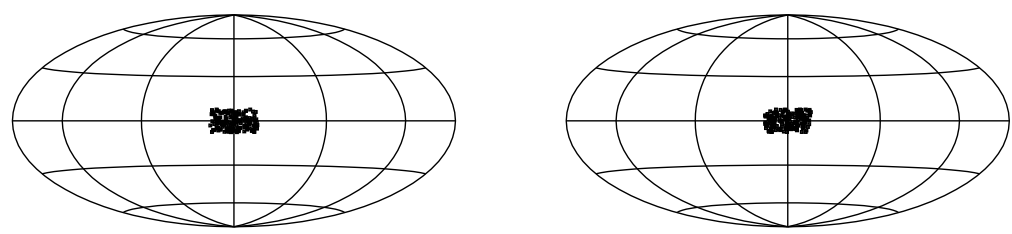

(c)
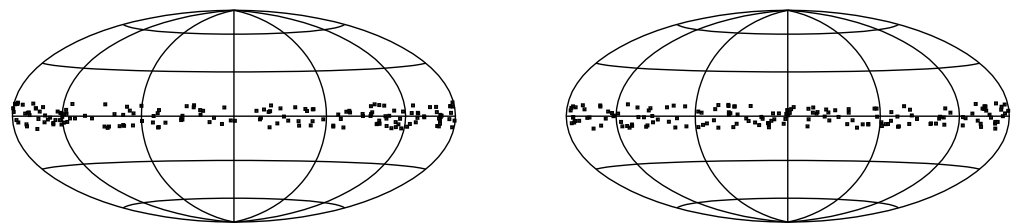

Figure 3: Aithoff-Hammer plots of Monte Carlo simulation with randomly generated sources restricted to small angular patches on the dome of the sky. (a) A single patch with half-width in latitude and longitude of 7.5 degrees. (b) Two patches on opposite sides of the sphere with the same angular width as (a). (c) A belt of the same angular width centered on the equator. The JM1 $\times 3$ statistic for each case was evaluated for 10,000 randomly generated trials. Distributions of the test statistic were in excellent agreement with the predicted distribution $\chi_{3}^{2}$ in each of these three cases, and indistinguishable from those of Figure 2. The angular distributions, being far less isotropic than that of the data (compare Figure 1), serve to verify independence of the statistic on the sky distribution.

extent in right ascension and declination (Figure 3). We also examined the statistic's distribution with $\vec{X}$ values restricted to two oppositely oriented clusters of the same size, and restricted to an equatorial belt of the same angular width. In each case 10, 000 Monte Carlo runs were made to generate the distributions. The distribution of each case was indistinguishable from the others, and the same as the predicted $\chi_{3}^{2}$.

\subsection{Likelihood Analysis}

The more sensitive method is likelihood analysis, a straightforward contest between model distributions. The likelihood $L$ is defined to be the product of the normalized distribution $f(z)$ evaluated at the points $z_{i}$ of the data: $L=\prod_{i} f\left(z_{i}\right)$.

The logarithm of the likelihood is maximized as a function of distribution param- 
eters. Twice the difference of maximized log-likelihoods $(2 T)$ between a factorized null model and a model containing correlation is distributed like $\chi_{m}^{2}$, where $m$ is the difference in the number of parameters of the models. The effects of parameters on statistical significance is thereby taken into account. As mentioned earlier, each study we present takes into account the distribution of the sample on the dome of the sky explicitly by taking $f(\vec{X})$ directly from the data. We checked the results by extensive Monte Carlo statistical simulations (described below). To create the most optimistic definition, we conservatively allowed the null hypothesis to be whatever is the best a posteriori champion for the null hypothesis. This is a conservative bias. Nine independent functional forms with many free parameters were explored to represent $h(\Omega)$. As for the correlated distribution $C(\Omega, \vec{X})$, we did not make extensive searches, but used a standard map and the map produced by the best null fit in the correlation ansatz of Kendall and Young, ${ }^{3}$ both of which are odd functions under $\beta \rightarrow-\beta$ representing odd-parity.

\section{Invariant Correlations}

Following $N R$ (a signal odd in $\beta$ ), for our first preliminary study we apply the oddparity Map $1 J M$ correlations to the full data set, and to cuts based on rotation measure $(R M)$. An odd parity signal was also reported earlier by $\operatorname{Birch}^{2}$ for a smaller 137 point data set and verified later by Kendall and Young. ${ }^{3}$ The motivation for cutting on $R M$ is that this is the only variable we have to give a symmetric and unbiased cut. A further, physics-based motivation ${ }^{17}$ appears in the Discussion.

Results for the full data set, 361 points, with Map 1 are reported in Table 1. In particular we find $n \rho_{1 \times 3}^{2}=11.15$. This indicates a statistically significant indication of correlation with this measure, with a $\mathrm{P}$-value of $1 \%$. The usual isotropic uncorrelated hypothesis is ruled out at $99 \%$ confidence level. If we exclude the extra points from Birch's sample, we are left with a 332 point data set for which $n \rho_{1 \times 3}^{2}=8.17$ $(P-$ value $=4.3 \%)$, which also shows evidence for correlation.

We now turn to cuts based on rotation measure $(R M)$, which we apply to the 332 point data set (excluding Birch's compilation since we did not have the RM for this set). We examined the regions of $R M<0$ (153 points) and $R M \geq 0$ (179 points) separately, which in an unbiased set would be a search between 2 alternatives. Sticking to the strict cuts, the region of $R M \geq 0$ does not show evidence of correlation: $n \rho_{1 \times 3}^{2}=5.37$. However we find that the region of $R M<0$ shows a highly significant Map 1 correlation: $n \rho_{3 \times 1, \text { odd }}^{2}=14.3\left(\mathrm{P}\right.$-Value $\left.=3 \times 10^{-3}\right)$. For those accustomed to Gaussian 2-sided statistics, this corresponds to a deviation at greater than the $3 \sigma$ level, or a $99.7 \%$ confidence level that the null does not fit the data.

The question may arise whether the point $R M=0$ is unique. Varying the cut location, a significant correlation is quite persistent for cuts retaining $R M<0$. By varying the cut in the region $R M<-20$ to $R M<7$, we find statistically significant ( $95 \%$ or better) rejection of the null hypothesis for each of the 27 ways to choose 
Table 1. Correlation test statistics $n \rho^{2}$ as defined by Jupp and Mardia, a scale and rotationally invariant trace of products of $1 \times 3$ correlation matrices, along with maximum log-likelihood test statistics $2 T$. $\mathrm{P}$-values are the probability of fluctuations in the null distribution to equal or exceed the data's statistic. Results are given for two different choices of mappings $\beta \rightarrow \vec{Y}(\Omega)$ discussed in text, with Map 1 and Map 3 corresponding to $\Omega_{1}=2 \beta$ and $\Omega_{3}=2 \beta+\nu \sin (2 \beta)$ respectively. Best fits to the null hypothesis selected Map 3 over Map 1. P-values indicated by $*$ use $\chi_{5}^{2}$.

\begin{tabular}{|c|c|c|c|c|c|}
\hline $\begin{array}{l}\text { Data } \\
\text { set }\end{array}$ & $\begin{array}{l}\text { no. of } \\
\text { points }\end{array}$ & $\begin{array}{l}\text { Map 1 } \\
n \rho^{2}(\mathrm{P})\end{array}$ & $\begin{array}{l}\text { Map 1 } \\
2 \mathrm{~T}(\mathrm{P}) \\
\end{array}$ & $\begin{array}{c}\text { Map 3 }(\nu=-1) \\
n \rho^{2}(\mathrm{P})\end{array}$ & $\begin{array}{l}\text { Map 3 } \\
2 \mathrm{~T}(\mathrm{P}) \\
\end{array}$ \\
\hline Full & 361 & $11.15(1.1 \%)$ & $8.40(3.8 \%)$ & $12.77(0.5 \%)$ & $11.22(1.1 \%)$ \\
\hline $\begin{array}{l}\text { Excluding } \\
\text { Birch }\end{array}$ & 332 & $8.17(4.3 \%)$ & $5.54(14 \%)$ & $10.10(1.8 \%)$ & $8.6(3.5 \%)$ \\
\hline $\begin{array}{l}\mathrm{RM}<0 \\
\text { Excluding } \\
\quad \text { Birch }\end{array}$ & 153 & $14.34(0.2 \%)$ & $11.90(0.8 \%)$ & $17.25(0.06 \%)$ & $17.26(0.06 \%)$ \\
\hline $\begin{array}{l}\mathrm{RM} \geq 0 \\
\text { Excluding } \\
\quad \text { Birch }\end{array}$ & 179 & $5.37(15 \%)$ & $4.62(20 \%)$ & $3.60(30 \%)$ & $0.44(93 \%)$ \\
\hline $\begin{array}{c}|R M-\overline{R M}|>6 \\
\text { Excluding } \\
\text { Birch }\end{array}$ & 265 & $16.62\left(0.5 \%^{*}\right)$ & $15.56\left(0.8 \%^{*}\right)$ & $22.66\left(0.04 \%^{*}\right)$ & $21.62\left(0.06 \% \%^{*}\right)$ \\
\hline
\end{tabular}

the cut. The number of points varies quite rapidly in this region. Meanwhile the statistic nicely follow proportionality to the number of points $n_{\text {cut }}$, that is $n_{\text {cut }} \rho_{\text {cut }}^{2} \approx\left(n_{\text {cut }} / n_{R M<0}\right) \times n_{R M<0} \rho_{R M<0}^{2}$. This is non-trivial and not consistent with fluctuations ( $\rho^{2}$ is a nonlinear function) but rather consistent with finite $\rho^{2}$ being an intrinsic property of the sample. From this observation one projects that statistical significance should be lost for cuts on $R M<-20$, due to decreasing the number of points. Cutting the positive side of the origin of $R M, \mathrm{P}$-values exceed 5 $\%$ for cut values of $R M_{\min }>7$.

The significant correlation for the set $R M<0$ might be misinterpreted. The question arises whether this might be due to a decorrelated strip in the complementary region $R M \geq 0$ (as opposed to something special about $R M<0$ ). Indeed the $R M$ distribution is not Gaussian but instead has a shifted mean $\overline{R M}=6$, with a big spike at the mean plus or minus $6-10$ units. The central spike region, occuring entirely outside the $R M<0$ region, appears to be uncorrelated. The balance of the data is then highly correlated for both positive and negative $R M$. This is quite a striking phenomenon. This effect is most clear in the likelihood analysis (below): but it is also visible with $n \rho_{1 \times 3, \text { odd }}^{2}=16.6, P-$ value $<8 \times 10^{-4}$ for the region excluding $\overline{R M} \pm 6$. In order to account for the 2 parameters needed in specifying the cut, namely the mean and the width of the excluded region, we conservatively use $\chi_{5}^{2}$ distribution to evaluate the probability, and find a $\mathrm{P}$-value of $5 \times 10^{-3}$. Again this is not very sensitive to the method of cut: a statistically significant rejection 
of the null hypothesis at $99 \%$ confidence level occurs for every one of the 13 ways to exclude $\overline{R M} \pm 5$ to $\overline{R M} \pm 18$.

\section{Likelihood Analysis and the Map}

A more specific method of testing isotropy is likelihood analysis. A typical null distribution for angular variables is the von Mises $(v M)$ form, of the type

$$
h(\Omega) f(\vec{X})=\text { constant } \times \exp (k \cos (\Omega)) f(\vec{X})
$$

The distribution has its maximum at $\Omega=0$ or $\Omega=\pi$ for $k>0$ or $k<0$, respectively. The location of the maximum can be translated by adding a parameter $\Omega \rightarrow \Omega-\delta$. As a generic ansatz odd in $\beta$, we used the Kendall and Young ${ }^{3}(K Y)$ ansatz for correlation: it is

$$
C(\beta, \vec{X})=\exp \{\mu \vec{X} \cdot \vec{s} \sin (\Omega)\}
$$

The exponential forms are inspired by statistics, rather than any deep physical considerations: they are exponentials of linear and bilinear functions of $\vec{X}$ and $\vec{Y}$. The correlation ansatz depends on 3 parameters: a measure of correlation $\mu$, constructed so that $C=1$ when $\mu=0$, and 2 parameters locating a normalized $\vec{s}$. Lines of constant probability are a linear relation between components of $\vec{Y}$ and $\cos (\gamma)$, where $\gamma$ is the polar angle between a source and $\vec{s}$. To compare correlation ${ }^{\mathrm{a}}$ we separately maximized the log-likelihoods $L_{2}$ (correlated fit) and $L_{1}$ (null fit), extracting the test statistic $2 T=2\left(L_{2}-L_{2}\right)$, which is distributed like $\chi_{3}^{2}$.

Applying the maximum likelihood analysis to the full data set of 361 points, one finds (Map 1) that $2 T=8.40$, with a $\mathrm{P}$-value of $3.8 \%$ (or $96 \%$ confidence level evidence of dependence). In this case the best fit parameters are $(k=-0.65)$ (null), $(k=-0.66, \mu=0.39)$ (correlated), with axis parameters $\vec{s}=\left[\left(1^{\mathrm{h}}, 16^{\mathrm{m}}\right) \pm\right.$ $\left.\left(2^{\mathrm{h}}, 0^{\mathrm{m}}\right), 40^{\circ} \pm 20^{\circ}\right]$.

Having found that correlation fits significantly better than a standard null, we further examined several different Maps attempting to improve the null fit. We investigated von Mises distributions depending on $\Omega_{2}=2 \beta-\delta$, where $\delta$ is an arbitrary parameter. Other maps are:

Map 3: $\quad \Omega_{3}=2 \beta+\nu \sin (2 \beta)$

Map 4: $\quad \Omega_{4}=2 \beta+\nu \sin (2 \beta)+\delta$

Map 5: $\quad \Omega_{5}=2 \beta+\nu \sin (2 \beta+\delta)$

Map 6: $\quad \Omega_{6}=2 \beta+\nu \cos (2 \beta)$

Map 7: $\quad \Omega_{7}=2 \beta+\nu \cos (2 \beta)+\delta$

This gives 7 linearly independent maps in all. We also used $\Omega_{1}$ in the bimodal von Mises combination $\delta c_{1} \exp \left[k_{1} \cos (2 \beta)\right]+(1-\delta) c_{2} \exp \left[k_{2} \cos (2 \beta-\pi)\right]$ which has 3 parameters $\delta, k_{1}, k_{2}$, and in the 2- parameter cardiod distribution ${ }^{18} \frac{1}{\pi}\left(1+k \cos \left(\Omega_{1}-\right.\right.$

aThe shifted $v M\left(\right.$ Loredo et $\left.\mathrm{al}^{8}\right), g_{\text {shifted }-\mathrm{vM}}(\Omega)=$ constant $\times \exp (k \cos (\Omega-\theta(\vec{X}))$, where $\theta(\vec{X})$ is some correlating function, is an inappropriate test for correlation. First, the model correlation is even-parity, and cannot fit an odd-parity correlation we study here. Second, the marginal $\Omega$ distribution becomes completely tied to the $\theta(\vec{X})$ distribution: a uniform $\theta(\vec{X})$ distribution, e.g., generates a flat $\Omega$ marginal distribution, incompatible with the data. 
$\delta)$ ). We have then nine independent functional ways trying to make the null proposal fit the data.

As a result we were able to improve the likelihood of the null fit significantly, with Map 3 producing by far the best fit, about $2.4(2 T=4.8)$ units of likelihood higher than the $v M$ distribution while using only one more parameter. This map (standard in "circular statistics" biological studies ${ }^{19}$ on the swimming of fishes, escape of salamanders etc.) makes a more flattop or sharply peaked distribution than the $v M$, as definitely required here. To check for correlation we compare the same map in the $K Y$ correlation (Eq. 2).

Remarkably the improved null fits generate an even greater signal of correlation. ${ }^{a}$ The results are summarized in Table 1; for completeness, parameters are listed in the text, while uncertainties are listed as cited. The full data set, 361 points, shows $2 L_{2}-2 L_{1}=2 T=11.22$, a $P$-value of $1 \%$, or more than $2 \sigma$ 's deviation from the expectations of an uncorrelated distribution. This is another statistically significant indication of dependence. Objectively, the uncorrelated isotropic assumption is sufficiently worse in fitting the data that it is significantly disfavored. The best fit parameters in this case are $(k=-0.63, \nu=-0.50)$ (null), $(k=-0.61, \nu=-0.67, \mu=$ $0.47)$ (correlated), with axis parameters, $\vec{s}=\left[\left(0^{\mathrm{h}}, 33^{\mathrm{m}}\right) \pm\left(1^{\mathrm{h}}, 40^{\mathrm{m}}\right), 34^{\circ} \pm 20^{\circ}\right]$. For the 332 point set, which excludes Birch's compilation, we find $2 T=8.6$, $(P-$ value $=3.5 \%)$, with best fit parameters $(k=-0.62, \nu=-0.58)$ null, $(k=$ $-0.60, \nu=-0.75, \mu=0.44)$ (correlated), with axis parameters, $\vec{s}=\left[\left(23^{\mathrm{h}}, 22^{\mathrm{m}}\right) \pm\left(1^{\mathrm{h}}, 40^{\mathrm{m}}\right), 29^{\circ} \pm 20^{\circ}\right]$. On the full data set the cut $R M<0$ yields very significant evidence disfavoring the null distribution by more than $3.5 \sigma$, a $\mathrm{P}$ - value of $6 \times 10^{-4}$. If we exclude the region $|R M-\overline{R M}| \leq 6$ we find a spectacularly large correlation with $2 T=21.62$ and parameters $(k=-0.60, \nu=-0.37)$ (null), $(k=-0.55, \nu=-0.86, \mu=0.79)$ (correlated) with axis parameters,

$$
\vec{s}=\left[\left(0^{\mathrm{h}}, 9^{\mathrm{m}}\right) \pm\left(1^{\mathrm{h}}, 0^{\mathrm{m}}\right),-1^{o} \pm 15^{o}\right] .
$$

While the measures of significance are standard, we also verified the likelihood difference P-values by Monte Carlo calculations comparing the $2 T$ value for the data where $\beta$ was generated randomly, in one method by shuffling and in another method using our best fit distribution to the measured data. The Monte Carlo distributions are in excellent agreement and shown in Figure 2.

\section{Discussion}

\subsection{Parameters}

\footnotetext{
${ }^{a}$ In order to give the null the advantages, a common conservative bias, we actually optimized all combinations of $\chi^{2}$ degrees of freedom and likelihood values of the null distribution as compared to the correlated case. For example, one can pit the single-parameter $v M$ with lower likelihood against the more parameter Map 3 hoping to improve the null's significance using $\chi_{4}^{2}$. It fails. For another example, we conservatively rejected a slightly better fit for the bimodal $v M$. By having more parameters it presents a weaker case for the null proposal in likelihood comparisons.
} 
The statistics of $2 T$ take parameters into account. The $P$-values represent probabilities for fluctuations from the null distribution to appear correlated when fitting with any values of the parameters whatsoever. The parameter $\mu$ represents a strength of correlation in the model ansatz, which is found to be relatively "of order unity" compared to the other parameters. The parameter $\vec{s}$ represents orientation of a normalized axis with 2 degrees of freedom on the dome of the sky. The likelihood analysis yields $\vec{s}$ parameters that tend to agree reasonably within errors with the axis $\vec{s}_{N R}$ extracted in $N R: \vec{s}_{N R}=\left(21 \pm 2 \mathrm{hrs}, 0^{\circ} \pm 20^{\circ}\right)$. Variation is expected, of course, given the substantial differences in statistical approach, and different data sets. The 2-quadrant procedure of Nodland and Ralston ${ }^{1}$ is quite distinct from both the likelihood analysis and invariant correlations, making the near coincidence of axis surely significant. Some proposals for the axis orientation have been made earlier ${ }^{20,21}$ and numerous theoretical models, ${ }^{13,22,23,24,25,26,27,28,29,30}$ interpretations ${ }^{31,32,33,34,35}$ or related issues ${ }^{17}$ have come up.

\subsection{Cuts}

As emphasized earlier, the entire data set is correlated. Yet the cuts on $R M$ produce such a significant result that they cannot be dismissed. It is known that $R M$ is correlated with position on the sky; thus $R M$ cuts may tend to select certain regions preferentially. Figure 1 shows visually only a small change in the angular distribution, however. Our procedures take into account the population of sources on the dome of the sky, so the results are not due to changes in this population. We verified this extensively, using $\beta$ values from the data shuffled and assigned randomly to Monte Carlo generated points on the dome of the sky. The angular regions of the Monte Carlo data could even be restricted to patches far less isotropic than the data without detectable change in our statistic's distribution, as discussed earlier in the section on Independence of Statistics on Sky Distribution. We reiterate, then, that the statistic does not depend on the angular distribution of the data; unevenness of the sky distribution does not cause a correlation. But for the question what is causing the signal, we cannot rule out the possibility that different sky regions might be better correlated than others. Resolving such questions would go beyond the scope of this paper.

\subsection{Other Methods}

If $\beta$ were an angle depending on the angular coordinate system, transformed under change of angular origin by mixing the components of $\vec{Y}$, then another statistic that might be examined is the mixed even-odd parity combination $n \rho_{2 \times 3}^{2}$. As emphasized in Ref. [16] this quantity is free from any dependence on angular origin. While useful for some purposes, the statistic is not appropriate for our study, because $\beta$ is already invariant under change of origin. Equally important, representations of different parity are mixed together in a non-linear way in $\rho_{2 \times 3}^{2}$. As a result of combining many degrees of freedom, the mixed parity $2 \times 3$ statistic is distributed with 6 
degrees of freedom $\left(\chi_{6}^{2}\right)$ compared to 3 degrees of freedom for the pure-parity $1 \times 3$ correlations. It follows that the statistical significance of a pure parity-odd (or pure parity-even) correlation will not be properly evaluated, but greatly underestimated, using $\rho_{2 \times 3}^{2}$. This has been a point of confusion in the literature, as Bietenholz and Kronberg ${ }^{4,5}(B K)$ dismissed Birch's claims on the basis of the mixed-parity $\rho_{2 \times 3}^{2}$ statistic, while simultaneously citing that Birch had a pseudovector effect. We also were misled at first, and in preliminary work we confirmed $B K$ 's calculations. Surprisingly, the negative $R M$ region and center- $R M$ deleted regions are so highly correlated that even on the basis of the mixed $n \rho_{2 \times 3}^{2}$ there is a strong signal: for the 131 points of $B K$ 's set with $R M<0, n \rho_{2 \times 3, M a p 1}^{2}=19.48$, a confidence level of $99.7 \%$ that the set is correlated. Consistent with all the other results, this data set definitely has highly significant odd correlations $n \rho_{3 \times 1 \text { odd }}^{2}=11.50$, P-Value $=9 \times 10^{-3}$. There may also be mixed or even correlations: $n \rho_{3 \times 1, \text { even }}^{2}=8.64$, (P-value $=3.5 \%)$; the existence of both explains the large $n \rho_{2 \times 3}^{2}$. Otherwise we did not find significant even $J M_{1 \times 3}$ correlations.

Another approach is to calculate the $1 \times 1$ correlations of polarization representations and sky position vectors projected into a subspace defined by a fixed axis. A similar (mixed parity) procedure was reported by Bietenholz, ${ }^{5}$ but the method unfortunately involves searching for an axis parameter. We studied this in the early stages, revealing large correlations when axes were fit. We have choosen not to make claims about that method here, due to the difficulty of accounting for the effects of a parameter search, but as a consistency check the result is meaningful.

More recent suggestions ${ }^{6,8}$ to check sensitivity to the statistical baselines used in $N R$ have already been incorporated in more sophisticated ways by our analysis. In another study Carroll and Field ${ }^{7}$ suggested a minimum chi-squared procedure, using the Euclidean angular distances from the mean. The concept of an "average angle" defined by the arithmetic mean is then introduced. As discussed by Batschelet ${ }^{19}$ (see also Mardia ${ }^{36}$, Fisher ${ }^{18}$ ) the "average angle" so defined has caused problems in many fields. It is a quantity which does not transform properly, but instead depends on the convention used in binning angles. The same goes for the rms deviation from the mean, and we cannot use such methods to obtain estimators of anything physical or statistical.

As a side note, we remark that even-odd symmetry is also useful for discussing the null distribution. More demanding than simple independence is requiring the marginal distribution to be an even function of $\beta$. We did not force this, because a variety of effects, from bias to sampling statistics, might cause the data to violate this symmetry, and the best fit null of any kind was our goal. It seems very significant, then, that an unbiased marginal $\beta$ distribution fit delivers the even sharply -peaked ansatz, which is another indication that the data is acting physically.

\subsection{Are There Loopholes?}

Granted that the data is surprising, a conservative interpretation is certainly pru-

dent. A feature of likelihood analysis, vigorously addressed earlier, is that there 
might exist some unknown and better-fitting null distribution we were unable to find. To close any loopholes one might ask for a further statistic not depending on distribution models. Now the likelihood analysis obtained a sharply peaked Map 3, with the parameter $\nu=-1$ indicated. Adopting this Map we recalculated the odd$1 \times 3$ correlations. Strongly consistent is that with Map 3 all correlations increase again. Specifically (Table 1), for the full data set (361 points) $n \rho_{3 \times 1}^{2}=12.77$; for the data excluding Birch's set (332 points) $n \rho_{3 \times 1}^{2}=10.10$; for the cut excluding the region $|R M-\overline{R M}| \leq 6$, then $n \rho_{3 \times 1}^{2}=22.60$ with the corresponding P-values of $5 \times 10^{-3}, 2 \times 10^{-2}$ and $3 \times 10^{-5}$ respectively. These particular P-values should be interpreted with care because prior information was used. Yet the more sensitive Map 3 correlations are useful because they are independent of any distribution model. At better than $99.95 \%$ confidence level, the results show that no null distribution exists to fit the data.

\subsection{Bias}

Let us address the possibility of some consistently imposed bias. We make no pretense to special qualifications but have done what we can. Regarding polarizations, besides using the data sets already mentioned, we also checked consistency using a newer 1988 compilation. ${ }^{15}$ These polarization measurements are largely independent, scattering by a few degrees when sources coincide, as expected. Redoing the entire analysis with these polarizations as "primary" for coincident sources, the statistics are hardly changed, and the null is again ruled out at the $99 \%$ to $99.9 \%$ confidence level. Regarding the galaxy axes, we also have an independent set from the work of Bietenholz. ${ }^{5}$ Indeed this entire set is described as independently prepared; polarizations were taken from the literature, or from Kronberg, and axes were fit anew from maps. Again the data reveals signals with P-values of $10^{-2}$ to $10^{-4}$ contradicting the null hypothesis. To be specific, the JM Map 1 correlation is not very sensitive: Bietenholz's set of 277 points gives $n \rho_{1 \times 3}^{2}=4.78$, a weak indication but not statistically significant. However likelihood analysis shows that this entire sample is correlated. The best null distribution is again the sharply peaked ansatz; the statistic $2 T_{M a p} 3=8.7$, giving a $P-$ value $=3.4 \%$. Axis parameters are gratifyingly in agreement with those of the other data sets. The strong correlation this set gives for $R M<0$ region was already mentioned. Perfectly consistent, deleting a strip centered on the mean also gives a big signal in this independent data set.

\subsection{VLA Data}

There remains a VLA-generated data set of only about 30 points created very recently. ${ }^{37}$ Should such data be included with the set we have analyzed? Unfortunately these data are not comparable; the set consists of different physical observables. A close examination shows that galaxy axes are not used, but selected small pieces of straight "jets" are substituted. Moreover the entire set is taken from a 
regime where Faraday rotation is small and generally ignored. Direct polarizations at high frequencies were then substituted for our polarization variable $\chi$. But our $\chi$ comes from actually measuring Faraday rotation at different frequencies, and it is physically unjustified to connect these to the high frequency VLA values. There is unfortunately no model-independent method to connect the two kinds of data.

Another question that has arisen is one of data "quality". While the VLA data are certainly of high quality for the unrelated purpose of seeing fine structure inside radio galaxies, there is every reason to believe that the data we use is of high quality for the statistical purpose we have. We are quite enthusiastic for the terrific potential of using both kinds of data in complementary ways, especially since there is at least one physical mechanism ${ }^{17}$ which is frequency dependent and could exploit the differences. Indeed this effect requires a frequency regime for Faraday rotation not to be negligible. Perhaps the large $R M$ cuts found associated with the correlations are related, an interesting question that requires further investigation.

\section{Conclusions}

In presenting a study of restricted scope, our conclusions are most crisply phrased in a negative sense: the null hypothesis of uncorrelated isotropy is not supported. On the basis of significance, it can be ruled out. By the nature of this study, one is constrained from concluding prematurely what the correlation found may represent. Under many separate statistical probes, the evidence against isotropy in the data is significant at $95 \%-99.99 \%$ (roughly $2-4 \sigma$ ) confidence levels.

This is not the first such finding, but just one more among a number of studies accumulating over the years. While no evidence of systematic bias is found, we strongly reiterate the possibility. Yet the persistence of the effect seems to indicate physical processes outside the framework which has been used to interpret the data conventionally. Associated with this behavior are persistent axis parameters concordant with the axis parameters found in Nodland and Ralston, ${ }^{1}$ and which subsequently have been found to coincide with the CMB dipole direction. ${ }^{20,21} \mathrm{Nev}-$ ertheless this is a new field and it would be premature to fix on a physical origin now. We therefore postpone more detailed conclusions, and recommend that phys-

ical models be used to suggest suitable directions of research. Local effects, while traditionally held to be under control, can potentially be ruled out with redshift information. Resources exist to generate cosmological radio data sets with many more points, and the time may be ripe for clever technological advances that could be revolutionary. New analysis combined with new data might tell us what is causing the effect.

\section{Acknowledgements}

We thank Borge Nodland, Hume Feldman, Doug McKay and G. K. Shukla for useful comments. Supported by DOE grant number 85 ER40214, the KU General Research Fund, the NSF-K*STAR Program under the Kansas Institute for Theoretical and 
Computational Science and DAE grant number DAE/PHY/96152.

\section{References}

1. B. Nodland and J. P. Ralston, Phys. Rev. Lett. 78 (1997), 3043; B. Nodland J. P. and Ralston, Phys. Rev. Lett. 79 (1997), 1958; B. Nodland and J. P. Ralston, astroph/9708114; B. Nodland and J. P. Ralston, astro-ph/9706126.

2. P. Birch, Nature 298 (1982), 451.

3. D. G. Kendall and A. G. Young, MNRAS 207 (1984), 637.

4. M. F. Bietenholz and P. P. Kronberg, Astrophysical Journal 287 (1984), L1-L2.

5. M. F. Bietenholz, (1984), M. Sc. Thesis, University of Toronto.

6. D. J. Eisenstein and E. F. Bunn, Phys. Rev. Lett. 79 (1997) (Comments), 1957.

7. S. M. Carroll and G. B. Field, Phys. Rev. Lett. 79 (1997), 2934.

8. T. J. Loredo, E. E. Flanagan and I. M. Wasserman, Phys. Rev. D56 (1997), 7057.

9. E. S. Phinney and R. I. Webster, Nature 301 (1983), 735.

10. J. P. Ralston and P. Jain, submitted to Physical Review D.

11. W. Eichendorf and M. Reinhardt, Astro. Sp. Sci. 61 (1979), 153

12. W. Eichendorf and M. Reinhardt, Ac. Cos. Zesc. 9 (1980), 7

13. S. M. Carroll, G. B. Field and R. Jackiw, Phys. Rev. D41 (1990), 1231.

14. M. Simard-Normandine, P. P. Kronberg and S. Button, Astrophysical Journal Suppl. $45(1981), 97$.

15. N. W. Broten, J. M. MacLeod and J. P. Vallee, Astrophysics and Space Science 141 (1988), 303.

16. P. E. Jupp and K. V. Mardia, Biometrika 67 (1980), 163.

17. J. P. Ralston, P. Jain and B. Nodland, Phys. Rev. Lett. 81 (1998), 26.

18. N. I. Fisher, Statistics of Circular Data (1993) (Cambridge)

19. E. Batschelet, Circular Statistics in Biology (1981), (London: Academic Press).

20. R. W. Kühne, Mod. Phys. Lett. A12 (1997), 2473.

21. R. N. Bracewell and V. R. Eschelmann, aps1997jun13_006 (1997).

22. D. V. Ahluwalia and T. Goldman, Mod. Phys. Lett. A 28 (1993), 2623.

23. C. H. Brans, Astrophysical Journal 197 (1975), 1.

24. R. B. Mann, J. W. Moffat and J. H. Palmer, Phys. Rev. Lett. 62 (1989), 2765; R. B. Mann and J. W. Moffat, Can. J. Phys. 59 (1981), 1730.

25. W.-T. Ni, Phys. Rev. Lett. 38 (1977), 301.

26. J. P. Ralston, Phys. Rev. D 51 (1995) 2018.

27. M. Sachs, Nuovo Cimento 111A (1997), 611.

28. B. W. Tolman and R. A. Matzner, Phys. Rev. D. 26 (1982), 2951.

29. C. M. Will, Phys. Rev. Lett. 62 (1989), 369.

30. C. Wolf, Phys. Lett. A 132 (1988), 151.

31. A. Dobado and A. L. Maroto, astro-ph/9706044.

32. R. Mansouri and K. Nozari, gr-qc/9710028.

33. J. W. Moffat, astro-ph/9704300

34. Y. N. Obhukov, V. A. Korotky, and F. W. Hehl, astro-ph/9705243.

35. G. C. Surpi and D. D. Harari, astro-ph/9709087

36. K. V. Mardia, Statistics of Directional Data, (1972). (London: Academic Press)

37. J. F. C. Wardle, R. A. Perley and M. H. Cohen, Phys. Rev. Lett. 79, (1997) 1801. 